\title{
On the Fučík Spectrum for Equations with Symmetries
}

\author{
Bernhard Ruf
}

Dedicated to Jean-Pierre Gossez on the occasion of his 65th birthday

\begin{abstract}
An overview over some aspects of the Fučík spectrum are given, in particular in the situation when the problem is invariant under some compact group action. Some recent results concerning the complexity of the Fučík spectrum are discussed, and some open problems are stated. In the final section, based on the mentioned structure of the Fučiík spectrum, a new multiplicity result for a related equation with asymptotic interference with the spectrum is given.
\end{abstract}

\section{Introduction}

In this article we first review some recent results on the structure of the so-called Fučik spectrum - a nonlinear spectrum associated with the Laplacian. Consider the following simple nonlinear elliptic equation:

$$
\left\{\begin{aligned}
-\Delta u & =\lambda u^{+}-\mu u^{-}, & & \text {in } \Omega \\
B u & =0, & & \text { on } \partial \Omega
\end{aligned}\right.
$$

where $\Omega \subset \mathbb{R}^{N}$ is a bounded domain, $u^{+}:=\max \{u, 0\}, u^{-}:=\max \{-u, 0\}$, with $\lambda, \mu \in \mathbb{R}$, and $B u=0$ denoting suitable homogeneous boundary conditions.

The Fučík spectrum is defined as the set

$$
\Sigma=\left\{(\lambda, \mu) \in \mathbb{R}^{2}: \text { (1.1) has a nontrivial solution }\right\}
$$

The study of such type of equations goes back to S. Fučík [17] and E.N. Dancer [11], who introduced this spectrum, first in the study of the corresponding ODE

$$
\left\{\begin{array}{l}
-u^{\prime \prime}=\lambda u^{+}-\mu u^{-},(0, \pi) \\
B u=0
\end{array}\right.
$$

For equation (1.2) the Fučík-spectrum can be completely determined. For instance, for periodic boundary conditions

$$
B u=0 \Longleftrightarrow u(0)=u(\pi), u^{\prime}(0)=u^{\prime}(\pi)
$$

1991 Mathematics Subject Classification. Primary 35J57; Secondary 49R05.

Key words and phrases. Nonlinear partial differential equations, nonlinear spectrum. 
one finds global branches $\Sigma_{k}$ departing from each linear eigenvalue $\lambda_{k}, k=0,1, \ldots$; first, there is the so-called "trivial part" of the Fučík spectrum:

$$
\Sigma_{0}=\left\{\left(\lambda, \lambda_{0}\right), \lambda \in \mathbb{R}\right\} \cup\left\{\left(\lambda_{0}, \mu\right), \mu \in \mathbb{R}\right\},
$$

where $\lambda_{0}=0$ is the lowest eigenvalue of $-\frac{d^{2}}{d x^{2}}$ with periodic boundary conditions; these values in the Fučík spectrum correspond to solutions of constant sign;

then there are the curves

$$
\Sigma_{k}:=\left\{(\lambda, \mu) \in \mathbb{R}^{2}: \frac{1}{\sqrt{\lambda}}+\frac{1}{\sqrt{\mu}}=\frac{1}{k \pi}=\frac{2}{\sqrt{\lambda_{k}}}\right\}, k=1,2,3, \ldots ;
$$

they are easily obtained, since $u^{+}$and $u^{-}$satisfy linear equations, whose solutions are explicit. The above formulas are then obtained by matching the derivatives of $u^{+}$and $u^{-}$.

These branches constitute together the complete Fučík-spectrum:

$$
\Sigma=\bigcup_{k \geq 0} \Sigma_{k}
$$

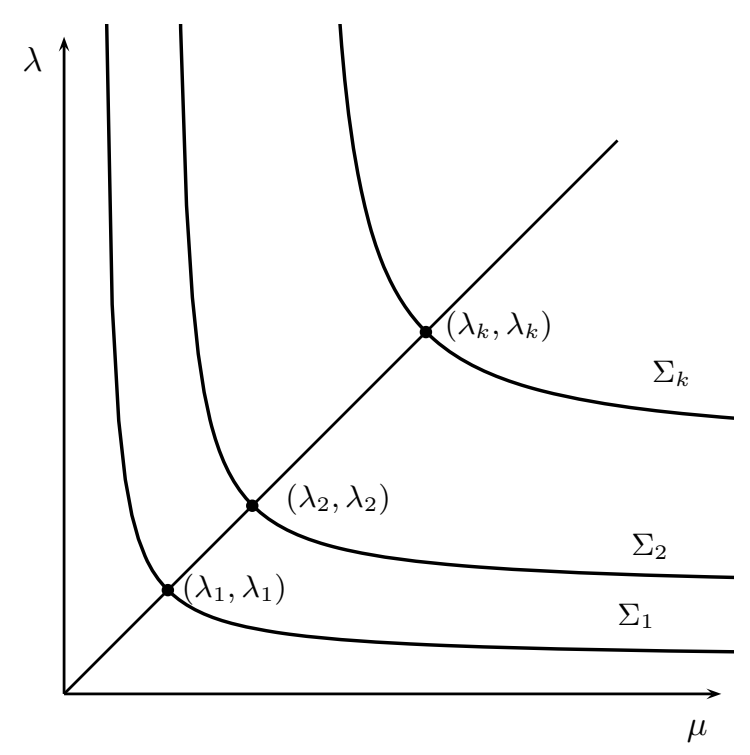

Figure 1: The Fučik spectrum

The Fučík spectrum has some important applications. First recall that the linear eigenvalues

$$
-u^{\prime \prime}=\lambda u, u(0)=u(1), u^{\prime}(0)=u^{\prime}(1),
$$

correspond to the (stationary) solutions of an oscillating system (say a string). 


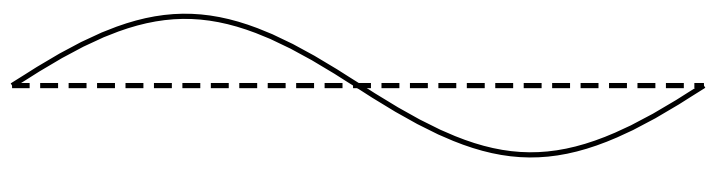

Figure 2: Linear oscillations

Similarly, the Fučík-spectrum corresponds to oscillations of an asymmetric system, where different reaction forces act in the positive and the negative direction.

$$
-u^{\prime \prime}=\lambda u^{+}-\mu u^{-}, u(0)=u(1), u^{\prime}(0)=u^{\prime}(1),
$$

Very interesting applications of this equation to the oscillations of suspension bridges have been given by Laser-McKenna in several influential papers, see e.g. $[20]$.

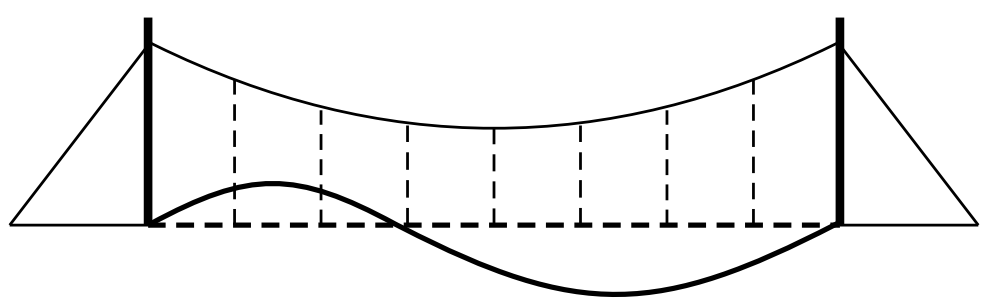

Figure 3: Nonlinear oscillations: the suspension bridge model

\section{The Fučík-spectrum for PDE's}

The Fučík-spectrum for corresponding partial differential equations is much more delicate, and much less is known. Consider the equation

$$
\left\{\begin{aligned}
-\Delta u & =\lambda u^{+}-\mu u^{-} \quad \text { in } \Omega \subset \mathbb{R}^{N} \\
u & =0 \quad \text { on } \partial \Omega
\end{aligned}\right.
$$

where $\Omega \subset \mathbb{R}^{N}$ is a bounded domain. Let $\lambda_{1}<\lambda_{2}<\lambda_{3}<\ldots$ denote the eigenvalues of $-\Delta$ with Dirichlet boundary condiitons. Much research has been done 
on this subject, see e.g. Gallouet-Kavian [18], Ruf [30], Lazer-McKenna [19], de Figueiredo-Gossez [15], Dancer, [13], and many more ... . However, despite many efforts, the results remain much less complete than in the ODE case. The following is known:

- $\Sigma$ is a closed set

- the (trivial) lines $\left\{\left(\lambda, \lambda_{0}\right), \lambda \in \mathbb{R}\right\}$ and $\left.\left\{\lambda_{0}, \mu\right), \mu \in \mathbb{R}\right\}$ belong to $\Sigma$

- $\Sigma$ does not contain points with: $\lambda<\lambda_{0}$ or $\mu<\lambda_{0}$

- in each square $\left(\lambda_{k-1}, \lambda_{k+1}\right)^{2}$ around $\left(\lambda_{k}, \lambda_{k}\right)\left(\lambda_{k}\right.$ may have multiplicity $m$ ), a continuum $C \subset \Sigma$ emanates from the point $\left(\lambda_{k}, \lambda_{k}\right) \in \Sigma$. $C$ is composed of a lower curve $C_{l o}$ and an upper curve $C_{u p}$; both curves are decreasing, and may be coincident (see Ruf [30], Gallouet-Kavian [18] for the situation of a simple eigenvalue $\lambda_{k}$, and Magalhães [22] and Schechter [32] for the case that $\lambda_{k}$ is a multiple eigenvalue.

- other points in $\Sigma \cap\left(\lambda_{k-1}, \lambda_{k+1}\right)^{2}$ can only lie between the curves $C_{l o}$ and $C_{u p}$; in particular, in the squares $\left(\lambda_{k-1}, \lambda_{k}\right)^{2}$ and $\left(\lambda_{k}, \lambda_{k+1}\right)^{2}$ there are no points of $\Sigma$ ).

- lowest part of the continuum $\Sigma_{1}$ departing from $\left(\lambda_{1}, \lambda_{1}\right)$, i.e. the "first nontrivial curve" in $\Sigma$. A global variational characterization of this set has been given by de Figueiredo-Gossez [15].

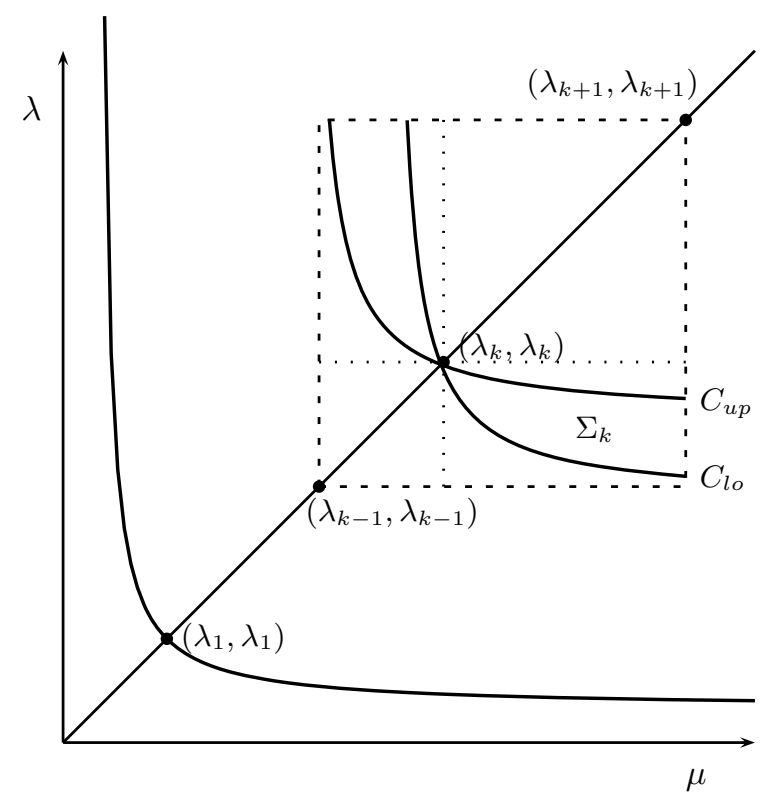

Figure 4: The Fučik spectrum near a higher eigenvalue 


\section{Generalizations of the Fučík spectrum to other type of equations}

The above results have been generalized in various directions, which we indicate below together with some bibliographic references.

\subsection{Second order equations with variable coefficients:}

$$
\left\{\begin{array}{l}
-\Delta u=m(x) u^{+}-n(x) u^{-}, \text {in } \Omega \\
u(x)=0, \partial \Omega
\end{array}\right.
$$

For the case $N=1$, we refer to Drábek [14], Arias-Campos [2], Rynne [31], ReichelWalter [29]; Alif-Gossez [1] considered the $N$-dimensional case, and with indefinite coefficients $m$ and $n$.

\subsection{Quasilinear equations with p-Laplacian:}

The analogue of the Fučík spectrum for the $p$-Laplacian takes the following form:

$$
\left\{\begin{array}{l}
-\Delta_{p} u=a\left(u^{+}\right)^{p-1}-b\left(u^{-}\right)^{p-1}, \text { in } \Omega \\
u(x)=0, \partial \Omega
\end{array}\right.
$$

The case $N=1$ was treated by Drábek [14]; in the $N$-dimensional case, Cuesta-de Figueiredo-Gossez [10] characterized the first curve in the Fučík spectrum, Perera [28] considered the general case (higher order branches), Arias-Campos-CuestaGossez treated the case with $a$ and $b$ variable $[\mathbf{3}]$ and indefinite [4].

\subsection{Equations with higher order differential operators:}

$$
\left\{\begin{array}{l}
-\Delta^{2} u=a u^{+}-b u^{-}, \text {in } \Omega \\
u(x)=\Delta u(x)=0, \partial \Omega
\end{array}\right.
$$

Campos-Dancer [7] studied the fourth-order ODE, Massa-Ruf [26] obtained results for the problem in $N$ dimensions, as a special case of a system of 2 nd order equations.

\subsection{Fučík-spectrum for elliptic systems:}

$$
\left\{\begin{array}{l}
-\Delta u=a v^{+}-b v^{-}, \text {in } \Omega \\
-\Delta v=c u^{+}-d u^{-} \\
u(x)=v(x)=0, \partial \Omega
\end{array}\right.
$$

This system of equation was studied by Massa-Ruf in [26]. A complete description of the Fučík spectrum for the corresponding system of ODE's was given, and in [27] the system of PDE's was studied. 


\section{Variational characterization of $\Sigma$}

In many applications it is very useful to have a variational characterization of the Fučík-spectrum. In general, this is not easy to obtain due to the asymmetric structure of the equation. We note however that de Figueiredo-Gossez [15] gave a global variational characterization of the first non-trivial curve of the Fučík spectrum. For other variational results see also Perera [28], and Massa [24].

It has been observed in $[\mathbf{1 6}]$ that if the equation has an additional symmetry, then a variational structure may be naturally available.

Consider again the ODE with periodic boundary conditions:

$$
\left\{\begin{array}{l}
-u^{\prime \prime}=\lambda u^{+}-\mu u^{-}, \text {in }[0,1) \\
u(0)=u(1), u^{\prime}(0)=u^{\prime}(1)
\end{array}\right.
$$

The corresponding functional is invariant under the $S^{1}$-action given by the timeshifts:

$$
T_{\theta} u(t)=u(t+\theta), \theta \in[0,1)
$$

Using the geometric $S^{1}$-index by $\mathrm{V}$. Benci $[6]$ one obtains for every $k \in \mathbb{N}$ a minimax-characterization of critical values $c_{k}, k \in \mathbb{N}$, which corresponds to the $k$-th Fučík eigenvalue, see de Figueiredo-Ruf [16]. In particular, this allows to give a variational characterization of the complete Fučík spectrum.

\section{The Fučík-spectrum $\Sigma \subset \mathbb{R}^{2}$ of $-\Delta$ on the torus}

Consider now equation (2.1) on the flat two-dimensional torus

$$
T^{2}=(0,1) \times(0, r)
$$

that is

$$
\left\{\begin{array}{l}
-\Delta u=\lambda u^{+}-\mu u^{-} \quad \text { in } \mathbb{R}^{2} \\
u(x, y)=u(x+1, y)=u(x, y+r),
\end{array} \quad \text { for all }(x, y) \in \mathbb{R}^{2} .\right.
$$

An important feature of this problem is its invariance under the compact group action

$$
\begin{aligned}
& g=(s, t) \in G=[0,1) \times[0, r): \\
& g \cdot[u(x, y)]:=u(x+s, y+t)
\end{aligned}
$$

Denoting by

$$
F(u):=-\Delta u-\left(\lambda u^{+}-\mu u^{-}\right),
$$

then $F$ is equivariant under the action of $G$, i.e.

$$
F(g \cdot u)=g \cdot F(u), \text { for all } g \in G .
$$

Note that the linear eigenvalues of $-\Delta$ on $T^{2}$ are explicit:

$$
\lambda_{k}:=\lambda_{i, j}=i^{2} 4 \pi^{2}+j^{2} 4 \pi^{2} / r^{2}, i, j=0,1,2, \ldots
$$


5.1. Explicit branches in $\Sigma$. Using the structure of the torus, we can prove:

THEOREM 5.1. From every eigenvalue $\left(\lambda_{k}, \lambda_{k}\right)$ emanates an explicit global curve

$$
\Sigma_{k}^{\operatorname{expl}} \subset \Sigma
$$

given by:

(i) if $k=0$, then the lines $\left(\lambda, \lambda_{0}\right)$ and $\left(\lambda_{0}, \mu\right)$ are in $\Sigma$;

(ii) if $k \geq 1$, then the curve

$$
\Sigma_{k}^{\operatorname{expl}}: \frac{1}{\sqrt{\lambda}}+\frac{1}{\sqrt{\mu}}=\frac{2}{\sqrt{\lambda_{k}}}
$$

belongs to $\Sigma$

Thus, the explicit Fučík branches on the torus $T^{2}$ look just like in Figure 1.

Proof. On $[0,1] \times[0, r]$, use the change of variables

$$
\left\{\begin{array}{l}
z=j x+\frac{k y}{r} \\
w=j x-\frac{k y}{r},
\end{array} \quad \text { with } k, j \in \mathbb{N}\right. \text { mutually prime }
$$

5.2. Variational branches in $\Sigma$. As we already mentioned, it is desirable to have a variational characterization of the Fučík spectrum. For this we need an index for the torus-group $T^{2}$. Such an index is available due to the work of W. Marzantowicz [23] and T. Bartsch [5], who introduced an index for general compact Lie-groups.

A general G-index. Let $G$ be a compact Lie group and A a separable metric G-space; the action of a group element $g \in G$ on an element $a \in A$ is denoted by $g \cdot a$. The fixed point set of $G$ on $A$ is $A^{G}:=\{a \in A \mid g \cdot a=a, \forall g \in G\}$.

First, one defines an index relative to $A^{G}$

$$
\gamma_{e}\left(A^{G}\right)=\left\{\begin{array}{l}
\inf \left\{k \geq 0 \mid\left[A^{G}, S^{n}\right]=* \text { for any } n \geq k\right\} \\
0, \text { if } A^{G}=\emptyset
\end{array},\right.
$$

where $\left[A^{G}, S^{n}\right]$ denotes the set of all homotopy classes of maps from $A^{G}$ to $S^{n}$ and * denotes the class of those homotopic to a constant.

Now, consider all representations $V$ of the group $G$ such that

there exists a $G-$ map $f: A \rightarrow V \backslash\{0\}$ where

- $\operatorname{dim}_{\mathbb{R}} V^{G}=\gamma_{e}\left(A^{G}\right), f\left(A^{G}\right) \subseteq V^{G} \backslash\{0\}$

- $\left.f\right|_{A^{G}}$ is not homotopic to a constant as a map into $V^{G} \backslash\{0\}$,

and define

$$
\gamma_{G}(A)=\inf \left\{\operatorname{dim}_{\mathbb{C}} V_{G} \mid V \text { as in }(5.1)\right\},
$$

where $V_{G}$ is the complement of $V^{G}$ in $V$.

Main properties of the $G$-index (see Marzantowicz [23], and Massa-Ruf [25]): 
Proposition 5.2. Let $A, B$ be $G$-metric spaces.

1) If there exists a $G$-equivariant map $\Phi: A \rightarrow B$ such that $\left.\Phi\right|_{A^{G}}$ is a homotopy equivalence between $A^{G}$ and $B^{G}$, then $\gamma_{G}(A) \leq \gamma_{G}(B)$.

2) In particular, if $\Phi: A \rightarrow B$ is a $G$-equivariant homeomorphism, then $\gamma_{G}(A)=\gamma_{G}(B)$.

3) If $V$ is an orthogonal representation of $G$ and $S(V)$ the unit sphere in $V$, then $\gamma_{G}(S(V))=\operatorname{dim}_{\mathbb{C}} V_{G}$ and $\gamma_{e}\left(S\left(V^{G}\right)\right)=\operatorname{dim}_{\mathbb{R}} V^{G}$.

In what follows, we will consider the natural action of the group $G=T^{2}$ on the space $H=H^{1}\left(T^{2}\right)$ : for $g=(s, t) \in T^{2}$ and $u=u(x, y) \in H$

$$
g \cdot u=u(x+s, y+t)
$$

Observe that the fixed point set $H^{G}$ consists of the constant functions in $H$, and hence it corresponds to the one-dimensional eigenspace of the eigenvalue $\lambda_{0}$.

Using this G-index, more precisely the $T^{2}$-index, we can prove

TheOREM 5.3. From each eigenvalue $\left(\lambda_{k}, \lambda_{k}\right), k=1,2, \ldots$, emanates a global branch of values

$$
\Sigma_{k}^{\mathrm{var}} \subset \Sigma
$$

which can be characterized variationally.

Proof. With the help of the above index, one proceeds in the usual manner to obtain critical values: for $\mu \geq 0$ one sets

$$
\begin{aligned}
J_{\mu}: \quad H^{1}\left(T^{2}\right) \rightarrow \mathbb{R} \\
\\
u \mapsto J_{\mu}(u)=\int_{T^{2}}|\nabla u|^{2}-\mu \int_{T^{2}}\left|u^{+}\right|^{2},
\end{aligned}
$$

and one defines the classes of sets

$$
\Gamma_{k}=\left\{A \subseteq \partial B: A \text { closed, } A G \text {-invariant; } \pm \phi_{0} \in A ; \gamma_{G}(A) \geq k\right\}
$$

(here $B$ denotes the $L^{2}$ unit ball in $H^{1}\left(T^{2}\right)$ ).

Then one sets

$$
\lambda_{k}(\mu)=\inf _{A \in \Gamma_{k}} \sup _{u \in A} J_{\mu}(u)
$$

By the following proposition the values $\lambda_{k}$ are critical values.

Proposition 5.4. For $k \geq 1, \mu \geq 0$, the values $\lambda_{k}(\mu)$ are well defined positive critical values for $J_{\mu}$.

Proof. The proof proceeds by the following steps:

1) $\left.J\right|_{\partial B}$ is $G$-invariant, and satisfies the PS-condition

2) for each $k \geq 1: \Gamma_{k} \neq \emptyset$, and $\lambda_{k}(\mu)$ is well-defined

3) $\lambda_{k}(\mu)$ is critical: let $A_{\varepsilon} \in \Gamma_{k}$ with

$$
\sup _{u \in A_{\varepsilon}} J_{\mu}(u)<\lambda_{k}(\mu)+\varepsilon,
$$


and suppose that $\lambda_{k}(\mu)$ is not critical: then by the G-equivariant deformation lemma in $\partial B$

$$
\sup _{u \in \eta\left(A_{\varepsilon}\right)} J_{\mu}(u)<\lambda_{k}(\mu)-\varepsilon
$$

where $\eta$ is an equivariant homeomorphism satifying $\eta\left( \pm \phi_{0}\right)= \pm \phi_{0}$; thus, $\eta\left(A_{\varepsilon}\right) \in$ $\Gamma_{k}$, and hence we have a contradiction.

To complete the proof of Theorem 5.3, note that critical points of $J_{\mu}$ at level $\lambda_{k}(\mu)$ correspond to nontrivial solutions in $H^{1}\left(T^{2}\right)$ of the equation

$$
-\Delta u=\left(\lambda_{k}(\mu)+\mu\right) u^{+}-\lambda_{k}(\mu) u^{-},
$$

and hence

$$
\left(\lambda_{k}(\mu)+\mu, \lambda_{k}(\mu)\right) \in \Sigma_{k}^{\mathrm{var}}
$$

Finally, note that $\mu=0 \Rightarrow \lambda_{k}(0)=\lambda_{k}$, and hence $\Sigma_{k}^{v a r}$ emanates from $\left(\lambda_{k}, \lambda_{k}\right)$.

5.3. The global picture. By subsections 5.1 and 5.2 we have the existence of an

- explicit global branch $\Sigma_{k}^{\text {expl }}, \mathrm{k}=1,2, \ldots$

- global variational branch $\Sigma_{k}^{\mathrm{var}}, \mathrm{k}=1,2, \ldots$

emanating from the same eigenvalue $\left(\lambda_{k}, \lambda_{k}\right)$. Thus it is natural to ask the

Question: do these two branches coincide?

Answer: for certain eigenvalues they do coincide initially.

Indeed, adapting the result of Magalhães [22] and using the group invariance, one has

Proposition 5.5. Let $\lambda_{k}$ be an eigenvalue associated to a two dimensional eigenspace. Then all points in $\Sigma \cap\left(\lambda_{k-1}, \lambda_{k+1}\right)^{2}$ lie on the curve $\Sigma_{k}^{\operatorname{expl}}$, given in Theorem 5.1.

From this we conclude that if $\lambda_{k}$ is an eigenvalue with two-dimensional eigenspace, then in the rectangle

$$
\left(\lambda_{k-1}, \lambda_{k+1}\right)^{2}
$$

the two curves $\Sigma_{k}^{\mathrm{expl}}$ and $\Sigma_{k}^{\mathrm{var}}$ coincide. 


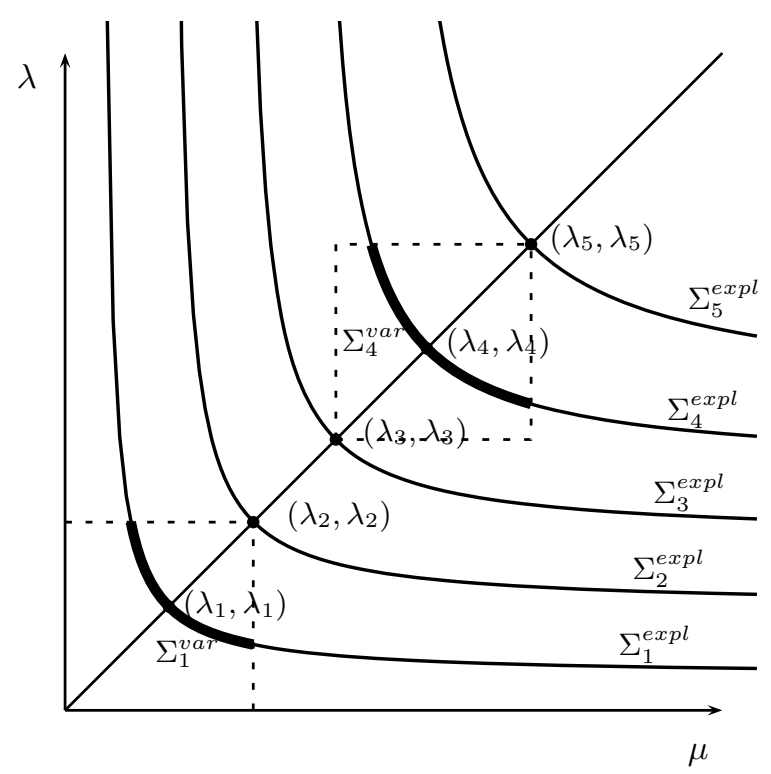

Figure 5: $\Sigma^{\text {expl }}$ and $\Sigma^{\text {var }}$ coincide near eigenvalues

Note that the explicit branches $\Sigma_{k}^{\operatorname{expl}}$ have asymptotes at

$$
\left(\frac{\lambda_{k}}{4},+\infty\right) \text { and }\left(+\infty, \frac{\lambda_{k}}{4}\right) ;
$$

this follows immediately from the formula $\frac{1}{\sqrt{\lambda}}+\frac{1}{\sqrt{\mu}}=\frac{2}{\sqrt{\lambda_{k}}}$.

On the other hand, we have:

TheOrem 5.6. All variational branches $\Sigma_{k}^{\mathrm{var}}$ tend asymptotically to $(0,+\infty)$ and $(+\infty, 0)$, respectively.

Proof. We construct sets $A_{\mu}$ with $G$-index

$$
\gamma_{G}\left(A_{\mu}\right) \geq k
$$

such that for $\mu$ large

$$
\sup _{u \in A_{\mu}} J_{\mu}(u)<\varepsilon .
$$

For this we start with a continuous function

$$
f(x)= \begin{cases}-\delta & \text { if } x \notin B_{\varepsilon}\left(x_{0}\right) \\ m & \text { if } x=x_{0}\end{cases}
$$

and such that

$$
\int_{T^{2}}|\nabla f|^{2} d x \rightarrow 0 \text { as } \varepsilon \rightarrow 0
$$

note that such a construction is impossible in dimension 1, but it is possible in dimensions $N \geq 2$. 
Next, we use the topological construction of "join" (more precisely, a $k$-fold join), and apply a topological result of Monica Clapp [8] to show that the resulting set $A_{\mu}$ satisfies indeed

$$
\gamma_{G}\left(A_{\mu}\right) \geq k
$$

Finally, one shows that:

$$
\sup _{A_{\mu}} J_{\mu} \rightarrow 0, \text { as } \mu \rightarrow \infty,
$$

by suitable estimates.

Hence, we conclude that many branch crossings must occur! Indeed: every explicit curve $\Sigma_{k}^{\text {expl }}$ gets crossed by all variational curves $\Sigma_{k}^{\text {var }}$ which start above it, i.e. by infinitely many curves.

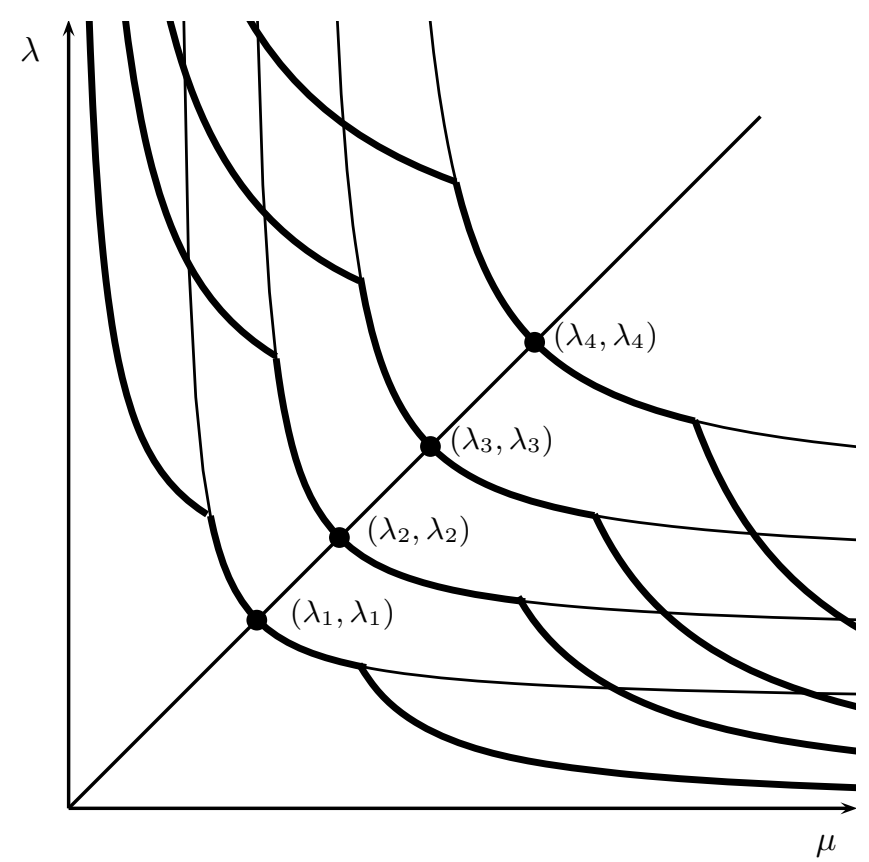

Figure 6: Branch crossings in the Fučik spectrum

5.4. Secondary bifurcations. In this section we study how the variational curves separate from the explicit branches.

THEOREM 5.7. On the first explicit branch $\Sigma_{1}^{\text {expl }}$ there exist infinitely many points of secondary bifurcation.

Proof. The classical tool for bifurcation is degree-theory. To treat the general case, we would need a $T^{2}$-equivariant degree theory. 
However, on the first branch $\Sigma_{1}^{e x p l}$ we can take advantage of the fact that the solutions depend just on one variable. Exploiting this symmetry, one can reduce the problem to a Neumann problem on the domain

$$
R:=(0,1 / 2) \times(0, r / 2)
$$

We can write this as the following equivalent problem: look for solutions of

$$
F(\mu, \lambda, u)=0
$$

where

$$
\begin{gathered}
F: \mathbb{R}^{2} \times H \rightarrow \mathbb{R} \times H: \\
F(\mu, \lambda, u)=\left(\|u\|_{L^{2}}^{2}-1, u-K\left[u+\lambda u+\mu u^{+}\right]\right) ;
\end{gathered}
$$

here $H=H^{1}(R) ; K=(-\Delta+I d)^{-1}: H \rightarrow H$

We are interested in bifurcations from known solution, i.e. solutions with $(\lambda+\mu, \lambda) \in$ $\Sigma_{1,0}^{\mathrm{expl}}$. We define a continuous function

$$
(0,+\infty) \ni \mu \mapsto\left(\lambda_{\mu}, u_{\mu}\right)
$$

such that

$$
\left(\lambda_{\mu}+\mu, \lambda_{\mu}\right) \in \Sigma_{1,0}^{\operatorname{expl}}
$$

and $u_{\mu}$ are the related explicit solutions with $\left\|u_{\mu}\right\|_{L^{2}}=1$. We have the following sufficient condition for bifurcation:

$$
0 \text { is a simple eigenvalue of the derivative } F_{(\lambda, u)}\left(\mu, \lambda_{\mu}, u_{\mu}\right)
$$

This is equivalent to the following condition: $\sigma=0$ is an eigenvalue with multiplicity 2 of

$$
\left\{\begin{array}{rlrl}
-\Delta v-\mu \chi_{u_{\mu}} v-\lambda_{\mu} v & =\sigma v & \text { in } R \\
v & =0 & \text { on } \partial R
\end{array},\right.
$$

where $\chi_{u_{\mu}}$ denotes the characteristic function of the set $\left\{u_{\mu}>0\right\}, \lambda_{\mu}$ is such that $\left(\lambda_{\mu}+\mu, \lambda_{\mu}\right) \in \Sigma_{1,0}^{\operatorname{expl}}$, and $u_{\mu}$ is the corresponding solution with $\left\|u_{\mu}\right\|_{L^{2}}=1$.

The spectrum of this equation can be characterized:

Lemma 5.8. For $\mu \rightarrow \infty$ there is a discrete sequence of values $\lambda_{j}(\mu)$ for which $\lambda=0$ is double eigenvalue, otherwise, $\sigma=0$ is simple eigenvalue.

We now can give the following

Interpretation: The variational branch $\Sigma_{1}^{v a r}$ initially follows the explicit branch $\Sigma_{1}^{\operatorname{expl}}$ (as seen above), and then, at the first branching point, it will follow the branch of secondary bifurcation (going asymptotically to zero). 


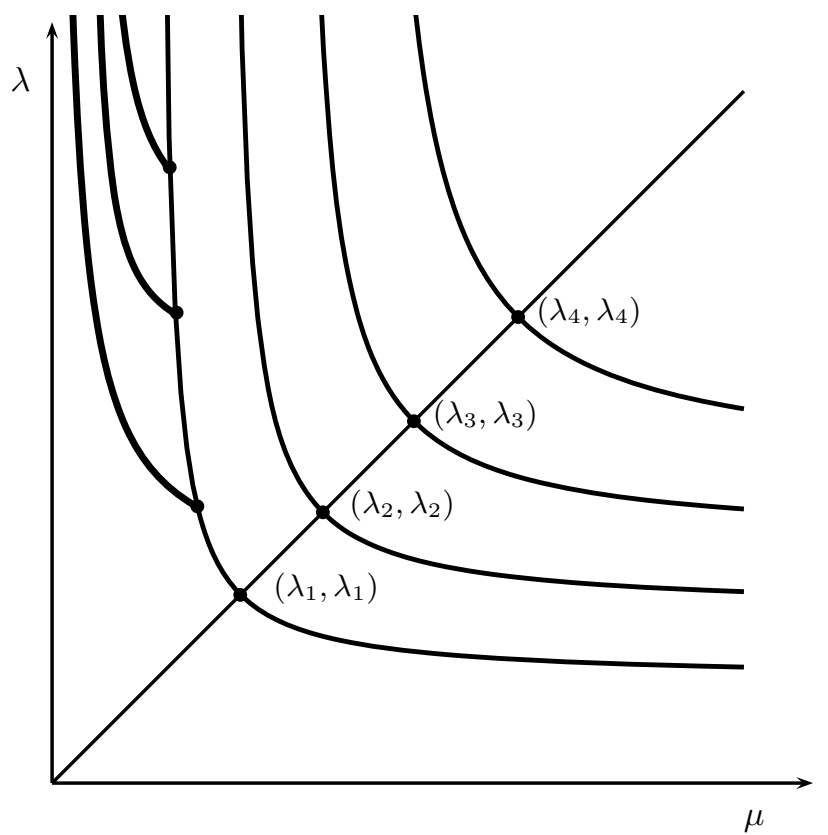

Figure 7: Secondary bifurcation of Fučík branches

Furthermore, we can also state the following:

THEOREM 5.9. The secondary bifurcations on the first branch $\Sigma_{1}$ are symmetry breaking.

Proof. The solutions on the first explicit curve depend on a single variable, and hence have a $S^{1}$-symmetry. On the other hand, the solutions on the secondary bifurcation branch break this symmetry (otherwise they would lie on $\Sigma_{1}^{e x p l}$ ), and hence their orbit is homeomorphic to the full group $T^{2}$.

\subsection{Open Problems.}

- give a global description of the complete Fučík spectrum

- are all Fučík-eigenvalues variational ?

In the following Figure 8 we give a possible global structure of the Fučíkspectrum which would permit that the whole Fučík spectrum is variational: as we know, the first variational branch $\Sigma_{1}^{v a r}$ initially coincides with the explicit curve $\Sigma_{1}^{\operatorname{expl}}$, until it encounters a bifurcation point, say $b_{1,1}$; here, the variational branch separates from the explicit curve, and tends asymptotically to zero. Also the second variational branch $\Sigma_{2}^{v a r}$ initially follows the explicit branch $\Sigma_{2}^{\text {expl }}$, bifurcates in $b_{2,1}$, and then meets the first branch in the point $b_{1,1}$ and follows from here the explicit branch $\Sigma_{1}^{e x p l}$, until it encounters the next bifurcation point $b_{1,2}$ where it separates again from the explicit branch and converges asymptotically to zero, and so on. We emphasize that this is just a possible structure, and in view of our results probably 
the simplest possible structure - however, the Fučík spectrum might also be much more complicated...

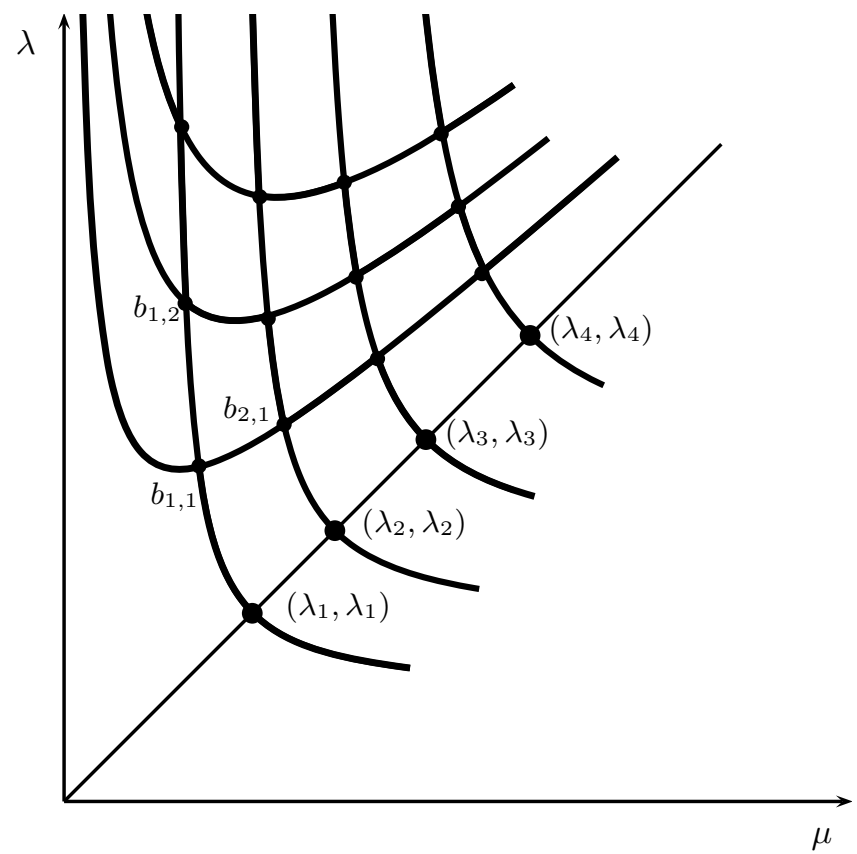

Figure 8: A possible global structure of the Fučík spectrum

\section{A multiplicity result for an equation with asymptotic interference with the spectrum}

In this section we prove a new multiplicity result for a forced equation of Fučík type. Consider the following equation

$$
\left\{\begin{array}{rl}
-\Delta u & =\alpha u^{+}-\beta u^{-}-t, \text { in } T^{2} \\
u & T^{2} \text {-periodic }
\end{array},\right.
$$

where $t>0$ is a given constant forcing term.

Note that equation (6.1) has the "trivial" solution $u=\frac{t}{\alpha}=: s_{t}$. We write $u=v+s_{t}$, and equation (6.1) as

$$
-\Delta v=\alpha\left(v+s_{t}\right)+(\alpha-\beta)\left(v+s_{t}\right)^{-}-t
$$

which is equivalent to

$$
\begin{aligned}
-\Delta v & =\alpha v+(\alpha-\beta)\left(v+s_{t}\right)^{-} \\
& =: \alpha v+\gamma\left(v+s_{t}\right)^{-}
\end{aligned}
$$


here $v=0$ corresponds to the trivial solution $u=s_{t}$. We look for nontrivial solutions of (6.2) and hence of (6.1). In this section we write the eigenvalues $\lambda_{k}$ repeated according to half of their multiplicity.

Theorem 6.1. Let $\alpha \in\left(\lambda_{k}, \lambda_{k+1}\right)$ be given. Then, for $\beta \in\left(0, \lambda_{1}\right)$ sufficiently small, equation (6.1) has at least $k$ non-trivial (orbits of) solutions.

\section{REMARK 6.2.}

1) The statement of this theorem is connected with the so-called Lazer-McKenna conjecture $[\mathbf{2 1}]$ which says that if a nonlinearity crosses asymptotically the first $k$ eigenfunctions, then the corresponding equation has at least $2 k$ nontrivial solutions. Indeed, the nonlinearity

$$
g(r):=\alpha r+\gamma\left(r+s_{t}\right)^{-}
$$

satisfies

$$
\lim _{r \rightarrow-\infty} \frac{g(r)}{r}=\alpha-\gamma=\beta \in\left(0, \lambda_{1}\right), \text { and } \lim _{t \rightarrow 0} \frac{g(r)}{t}=\alpha>\lambda_{k},
$$

and so it crosses indeed the first $k$ non-trivial eigenvalues, see also Remark 6.5 below.

2) It is interesting that a constant forcing term has the effect of generating multiple solutions. It is this effect which plays a fundamental role in Lazer-McKenna's treatment of the suspension bridge model [20], where the constant force given is a constant wind blowing against the bridge.

Proof. Consider the functional

$$
J(v)=\frac{1}{2} \int_{T^{2}}|\nabla v|^{2} d x-\frac{\alpha}{2} \int_{T^{2}}|v|^{2} d x+\frac{\gamma}{2} \int_{T^{2}}\left|\left(v+s_{t}\right)^{-}\right|^{2} d x
$$

Clearly, the functional $J: H:=H^{1}\left(T^{2}\right) \rightarrow \mathbb{R}$ is a $C^{1}$-functional, and it is invariant under the torus group $T^{2}$. We apply the following theorems which are a generalizations of Theorems 8 and 9 in the article [9] by D.C. Clark, generalized to the context of the torus group $T^{2}$ :

Theorem 6.3. Suppose that $I: H \rightarrow \mathbb{R}$ is a $C^{1}$-functional which is invariant under the torus group $G=T^{2}$, and satisfying the Palais-Smale condition. Let $\gamma_{G}$ denote the $G$-index defined in section 5.2, and let

$$
c_{m}(I)=\inf _{\gamma_{G}(A) \geq m} \sup _{u \in A} I(u)
$$

Then, if $-\infty<c_{m}<0$ the critical set $K_{c_{m}}$ is non-empty and compact. Moreover, if $-\infty<c_{m}(I)=c_{n}(I)=c<0$, then $\gamma\left(K_{c}\right) \geq n-m-1$.

TheOREM 6.4. Suppose in addition to the hypotheses of Theorem 6.3 that $I(0)=0$. Let $I_{a}:=\{u \in E \mid I(u) \leq a\}$ denote the sublevel set of $I$ at level a. Then, for any integer $m$ with

$$
\lim _{a \rightarrow 0^{-}} \gamma\left(I_{a}\right) \geq m>\lim _{a \rightarrow-\infty} \gamma\left(I_{a}\right)
$$

there exists at least one critical point $x_{m}$ (and hence a critical orbit $G x_{m}$ ) such that

$$
c_{m}(I)=\inf _{\gamma_{G}(A) \geq m} \sup _{x \in A} I(u)=I\left(x_{m}\right)
$$


The functional $J$ defined in (6.3) is invariant under the torus group $G=T^{2}$. Furthermore, it satisfies the (PS)-condition. Indeed, suppose that $\left(v_{n}\right) \subset E$ is a (PS)-sequence, i.e. such that

$$
\left|J\left(v_{n}\right)\right|=\left.\left|\frac{1}{2} \int_{T^{2}}\right| \nabla v_{n}\right|^{2}-\frac{\alpha}{2} \int_{T^{2}}\left|v_{n}\right|^{2}+\frac{\gamma}{2} \int_{T^{2}}\left|\left(v_{n}+s_{t}\right)^{-}\right|^{2} \mid \leq c
$$

and

$$
J^{\prime}\left(v_{n}\right)[\varphi]=\int_{T^{2}} \nabla v_{n} \nabla \varphi-\alpha \int_{T^{2}} v_{n} \varphi-\gamma \int_{T^{2}}\left(v_{n}+s_{t}\right)^{-} \varphi=\varepsilon_{n}(\varphi) \rightarrow 0
$$

We first show that $v_{n}$ is bounded in $H$. Assume to the contrary that $\left\|v_{n}\right\| \rightarrow \infty$. Dividing (6.6) by $\left\|v_{n}\right\|$ and setting $\tilde{v}_{n}:=\frac{v_{n}}{\left\|v_{n}\right\|}$ we have (for a subsequence) $\tilde{v}_{n} \rightarrow \tilde{v}$ in $H$ and $\tilde{v}_{n} \rightarrow \tilde{v}$ strongly in $L^{p}, p \geq 1$. Choosing $\varphi=v_{n}$ in (6.7) and subtracting it from $2 J\left(v_{n}\right)$ we get

$$
\left|\gamma \int_{T^{2}}\left(v_{n}+s_{t}\right)^{-}\right| \leq c+\varepsilon_{n}\left\|v_{n}\right\| .
$$

Dividing by $\left\|v_{n}\right\|$ we get in the limit

$$
\int_{T^{2}} \tilde{v}^{-}=0
$$

Next, we choose $\varphi=1$ in (6.7) and divide by $\left\|v_{n}\right\|$

$$
\frac{1}{\left\|v_{n}\right\|} J^{\prime}\left(v_{n}\right)[1]=-\alpha \int_{T^{2}} \tilde{v}_{n}-\gamma \int_{T^{2}}\left(\tilde{v}_{n}+\frac{s_{t}}{\left\|v_{n}\right\|}\right)^{-}=\frac{1}{\left\|v_{n}\right\|} \varepsilon_{n}(1) \rightarrow 0 ;
$$

thus we see that $\int_{T^{2}} \tilde{v}_{n} \rightarrow 0$ and by (6.8) that $\int_{T^{2}}\left|\tilde{v}_{n}\right| \rightarrow 0$, and hence $\tilde{v}=0$. Finally, by (6.7)

$$
\frac{1}{\left\|v_{n}\right\|} J^{\prime}\left(v_{n}\right)\left[\tilde{v}_{n}\right]=\int_{T^{2}} \nabla \tilde{v}_{n} \nabla \tilde{v}_{n}=\alpha \int_{T^{2}}\left|\tilde{v}_{n}\right|^{2}+\gamma \int_{T^{2}}\left(\tilde{v}_{n}+\frac{s_{t}}{\left\|v_{n}\right\|}\right)^{-} \tilde{v}_{n} \rightarrow 0
$$

we get a contradiction, since $1=\left\|\tilde{v}_{n}\right\|^{2}=\int_{T^{2}}\left|\nabla \tilde{v}_{n}\right|^{2}+\left|\tilde{v}_{n}\right|^{2}$. Thus, $\left\{v_{n}\right\}$ is bounded. From this follows in a standard way that there is a convergent subsequence in $E$.

Next, we verify condition (6.5).

Claim: If $\alpha>\lambda_{k}$, then $\lim _{a \rightarrow 0^{-}} \gamma_{G}\left(J_{a}\right) \geq k$.

Let $H_{k}$ be the $2 k$-dimensional space spanned by the eigenfunctions corresponding to the eigenvalues $\lambda_{1}, \ldots, \lambda_{k}$. We show that the sublevel set $J_{a}$ contains a $T^{2}$-invariant set homeomorphic to a $2 k$-dimensional sphere, for some $a>0$. Indeed, since on the $2 k$-dimensional space $H_{k}$ all norms are equivalent, there exists a constant $c_{k}$ depending only on $k$ such that $\|v\|_{\infty} \leq c(k)\|v\|_{L^{2}}$ for all $v \in H_{k}$, and hence $\int_{T^{2}}\left|\left(v+s_{t}\right)^{-}\right|^{2}=0$ for $v \in B_{\delta} \cap H_{k}$ with $\delta>0$ sufficiently small, where $B_{\delta}$ is the $L^{2}$-ball of radius $\delta$. For this $\delta$ we have

$$
\begin{aligned}
J\left(v_{n}\right) & =\frac{1}{2} \int_{T^{2}}|\nabla v|^{2}-\frac{\alpha}{2} \int_{T^{2}}|v|^{2}+\frac{\gamma}{2} \int_{T^{2}}\left|\left(v+s_{t}\right)^{-}\right|^{2} \\
& \leq \frac{1}{2}\left(\lambda_{k}-\alpha\right) \int_{T^{2}}|v|^{2} \leq a, \text { for all } v \in B_{\delta} \cap H_{k}
\end{aligned}
$$

for some $a<0$. This implies that $J_{a}$ contains a set of index $\geq k$, and hence $\gamma_{G}\left(J_{a}\right) \geq k$.

Claim: If $\beta \in\left(0, \lambda_{1}\right)$ sufficiently small, then $\lim _{a \rightarrow-\infty} \gamma_{G}\left(J_{a}\right)=0$. 
First note that by the characterization (5.2)

$$
\sup _{u \in A}\left[\frac{1}{2} \int_{T^{2}}|\nabla u|^{2}-\frac{\mu}{2} \int_{T^{2}}\left|u^{+}\right|^{2}-\frac{\lambda_{1}(\mu)}{2} \int_{T^{2}}|u|^{2}\right] \geq 0,
$$

for all $A$ with $\gamma(A) \geq 1$. To match this statement with the notation in the current section, we write $\alpha=\mu+\lambda_{1}(\mu)$, and get after collecting differently the terms

$$
\sup _{u \in A}\left[\frac{1}{2} \int_{T^{2}}|\nabla u|^{2}-\frac{\alpha}{2} \int_{T^{2}}|u|^{2}+\frac{\mu}{2} \int_{T^{2}}\left|u^{-}\right|^{2}\right] \geq 0
$$

hence, setting $\beta(\mu):=\alpha(\mu)-\mu=\lambda_{1}(\mu)$, we have that $(\alpha(\mu), \beta(\mu))$ lies on the first non-trivial Fučík curve.

Choose now

$$
\beta=\beta(\mu)-\delta=\alpha(\mu)-\mu-\delta=(\alpha(\mu)-\delta)-\mu,
$$

for some small $\delta>0$ (such that $\beta>0$ ). Set $\alpha=\alpha(\mu)-\delta$ and $\gamma=\mu$ in (6.3), and consider the sublevel set

$$
\begin{aligned}
J_{-n}= & \{u \in H \mid J(u) \leq-n\}=\left\{u \mid \frac{1}{n} J(u) \leq-1\right\} \\
= & \left\{u \mid v:=\frac{u}{\sqrt{n}}\right. \text { satisfies } \\
& \left.J^{(n)}(v):=\frac{1}{2} \int_{T^{2}}|\nabla v|^{2}-\frac{\alpha}{2} \int_{T^{2}}|v|^{2}+\frac{\gamma}{2} \int_{T^{2}}\left|\left(v+\frac{s_{t}}{\sqrt{n}}\right)^{-}\right|^{2} \leq-1\right\}
\end{aligned}
$$

We now show that for any set $A$ with $\gamma(A) \geq 1$ there exists $n_{0}$ with

$$
\sup _{v \in A} J^{(n)}(v) \geq-\frac{c}{n}, \text { for all } n \geq n_{0}
$$

Indeed,

$$
\begin{aligned}
\sup _{v \in A} J^{(n)}(v) & =\sup _{v \in A}\left[\frac{1}{2} \int_{T^{2}}|\nabla v|^{2}-\frac{\alpha}{2} \int_{T^{2}}|v|^{2}+\frac{\gamma}{2} \int_{T^{2}}\left|\left(v+\frac{s_{t}}{\sqrt{n}}\right)^{-}\right|^{2}\right] \\
& =\sup _{A}\left[\frac{1}{2} \int_{T^{2}}|\nabla v|^{2}-\frac{\alpha(\mu)}{2} \int_{T^{2}}|v|^{2}+\frac{\delta}{2} \int_{T^{2}}|v|^{2}+\frac{\mu}{2} \int_{T^{2}}\left|\left(v+\frac{s_{t}}{\sqrt{n}}\right)^{-}\right|^{2}\right]
\end{aligned}
$$

We estimate

$$
\begin{aligned}
\int_{T^{2}}\left|\left(v+\frac{s_{t}}{\sqrt{n}}\right)^{-}\right|^{2} & =\int_{\left[v \leq-\frac{s_{t}}{\sqrt{n}}\right]}\left|v+\frac{s_{t}}{\sqrt{n}}\right|^{2} \\
& \geq \int_{\left[v \leq-\frac{s_{t}}{\sqrt{n}}\right]}|v|^{2}+2 \int_{\left[v \leq-\frac{s_{t}}{\sqrt{n}}\right]} v \frac{s_{t}}{\sqrt{n}} \\
& \geq \int_{T^{2}}\left|v^{-}\right|^{2}-\int_{\left[-\frac{s_{t}}{\sqrt{n}} \leq v \leq 0\right]}|v|^{2}-2 \int_{T^{2}}\left|v^{-}\right| \frac{s_{t}}{\sqrt{n}} \\
& \geq \int_{T^{2}}\left|v^{-}\right|^{2}-\frac{s_{t}^{2}}{n}\left|T^{2}\right|-2 \frac{s_{t}}{\sqrt{n}} \int_{T^{2}}|v|+\frac{\delta}{2} \int_{T^{2}}|v|^{2} \\
& \geq \int_{T^{2}}\left|v^{-}\right|^{2}-\frac{c}{n} .
\end{aligned}
$$


Thus, we obtain by $(6.11)$

$$
\begin{aligned}
\sup _{v \in A} J^{(n)}(v) & \geq \sup _{u \in A}\left[\frac{1}{2} \int_{T^{2}}|\nabla u|^{2}-\frac{\alpha}{2} \int_{T^{2}}|u|^{2}+\frac{\mu}{2} \int_{T^{2}}\left|u^{-}\right|^{2}\right]-\frac{c}{n} \\
& \geq-\frac{c}{n}, \text { for any } A \text { with } \gamma_{G}(A) \geq 1 .
\end{aligned}
$$

This implies that there does not exist any set $A$ with $\gamma_{G}(A) \geq 1$ such that $A \subseteq$ $\lim _{n \rightarrow \infty} J_{-1}^{(n)}$. Thus, $\lim _{a \rightarrow-\infty} \gamma_{G}\left(J_{a}\right)=0$

From these claims the statements of Theorem 6.1 follow immediately by Theorem 6.4.

REMARK 6.5. Note that the assumption $\beta<\beta(\mu)$ in (6.12) says that the nonlinearity in fact must cross the $k$ variational Fučík eigenvalue branches $\Sigma_{j}^{v a r}, j=$ $1, \ldots, k$.

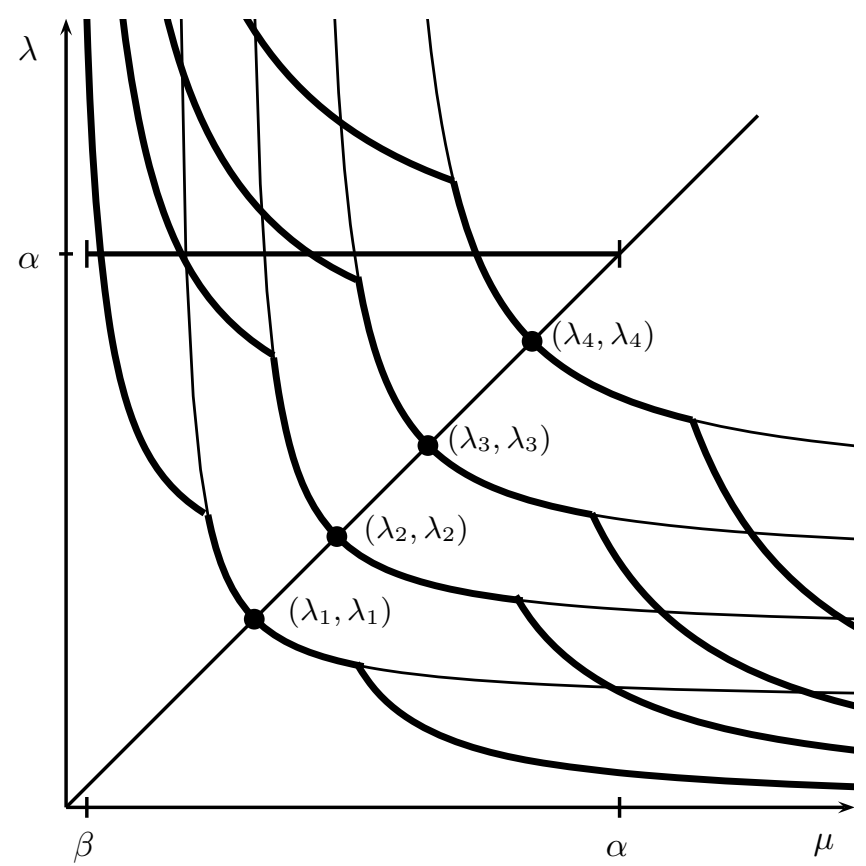

Figure 9: Asymptotic crossing of variational Fučik branches

\section{References}

[1] Alif, M.; Gossez, J.-P. On the Fucik spectrum with indefinite weights. Differential Integral Equations 14 (2001), no. 12, 1511-1530.

[2] Arias, M.; Campos, J. Fucik spectrum of a singular Sturm-Liouville problem, Nonlinear Anal. 27 (1996), no. 6, 679-697.

[3] Arias, M.; Campos, J.; Cuesta, M.; Gossez, J.-P. Asymmetric elliptic problems with indefinite weights, Ann. Inst. H. Poincaré Anal. Non Linéaire 19 (2002), no. 5, 581-616. 
[4] Arias, M.; Campos, J.; Cuesta, M.; Gossez, J.-P. An asymmetric Neumann problem with weights, Ann. Inst. H. Poincaré Anal. Non Linéaire 25 (2008), no. 2, 267-280.

[5] Bartsch, Thomas. Borsuk-Ulam theorems, the Lusternik-Schnirelmann category and index theories, World Congress of Nonlinear Analysts '92, Vol. I-IV (Tampa, FL, 1992), 2135-2146, de Gruyter, Berlin, 1996.

[6] Benci, Vieri. A geometrical index for the group $S^{1}$ and some applications to the study of periodic solutions of ordinary differential equations, Comm. Pure Appl. Math. 34 (1981), no. 4, 393-432.

[7] Campos, J.; Dancer, E.N. On the resonance set in a fourth-order equation with jumping nonlinearity, Differential Integral Equations 14 (2001), no. 3, 257-272.

[8] Clapp, Mónica. Borsuk-Ulam theorems for perturbed symmetric problems, Proceedings of the Third World Congress of Nonlinear Analysts, Part 6 (Catania, 2000). Nonlinear Anal. 47 (2001), no. 6, 3749-3758.

[9] Clark, David C. A variant of the Lusternik-Schnirelman theory, Indiana Univ. Math. J. 22 $(1972 / 73), 65-74$.

[10] Cuesta, M.; de Figueiredo, D.; Gossez, J.-P. The beginning of the Fucik spectrum for the p-Laplacian, J. Differential Equations 159 (1999), no. 1, 212-238.

[11] Dancer, E. N. On the Dirichlet problem for weakly non-linear elliptic partial differential equations, Proc. Roy. Soc. Edinburgh Sect. A 76, (1976/77), no. 4, 283-300.

[12] Dancer, E. N. Remarks on jumping nonlinearities, Topics in nonlinear analysis, 101-116, Progr. Nonlinear Differential Equations Appl., 35, Birkhäuser, Basel, 1999.

[13] Dancer, E.N., Some resutls for jumping nonlinearities 19 (2002) (2), 221-235.

[14] Drábec, P., Solvability and Bifurcations of Nonlinear Equations, Pitman Research Notes in Mathematics 264 (1992)

[15] de Figueiredo, D.G., Gossez, J.-P., On the first curve of the Fučik spectrum of an elliptic operator, Differential Integral Equations 7 (5-6) (1994), 1285-1302.

[16] de Figueiredo, D.G., Ruf, B., On the periodic Fučík spectrum and a superlinear SturmLiouville equation, Proc. Roy. Soc. Edinburgh, Sect. A, 123 (1) (1993), 95-107.

[17] Fučík, S., Boundary value problems with jumping nonlinearities, Časopis Pěst. Mat. 101 (1) (1976) 69-87

[18] Gallouët, T., Kavian, O., Résultats d'existence et de non-existence pour certain problìes demi-linéaires à l'infini, Ann. Fac. Sci. Toulouse Math. (5) 3 1981, 201-246, (1982)

[19] Lazer, A., McKenna, P.J., Multiplicity of solutions of nonlinear boundary value problems with nonlinearities crossing several higher eigenvalues, J. reine angew. Math. 368 (1986), 184-200.

[20] Lazer-McKenna, Large scale oscillatory behaviour in loaded asymmetric systems, Ann. Inst. H. Poincaré Anal. Non Linéaire 4 (3) (1987), 243-274.

[21] Lazer, A., McKenna, P.J., Multiple solutions of two point boundary value problems with jumping nonlinearities, J. Diff. Equ. 59 (2) (1985), 266-281.

[22] Magalhaes, C.A., Semilinear elliptic problem with crossing of multiple eigenvalues, Comm. Partial Differential Equations 15 (9) (1990), 1265-1292.

[23] Marzantowicz, W., A Brosuk-Ulam theorem for orthogonal $T^{k}$ and $Z_{p}^{r}$ actions and applications, J. Math. Anal. Appl. textbf137 (1) (1989), 99-121.

[24] Massa, E., On a variational characterization of a part of the Fučík spectrum of the Laplacian and a superlinear Sturm-Liouville equation, Proc. Royal Soc. Edinburgh Sect. A 134 (2004) (3) (2004), 557-577.

[25] Massa, E., Ruf, B., On the Fučík spectrum of the Laplacian on a torus, J. Funct. Analysis 256 (2009), 1432-1452

[26] Massa, E., Ruf, B., A global characterization of the Fučík spectrum for a system of ordinary differential equations, J. Diff. Equ. 234 (2007) (1), 311-336.

[27] Massa, E., Ruf, B., On the Fučík spectrum for elliptic systems, Top. Methods Nonlinear Analysis 27 (2) (2006), 195-228.

[28] (2004) Perera, K., On the Fučik spectrum of the p-Laplacian, NoDEA Nonlinear Diff. Equ. Appl. 11 (2) (2004), 259-270.

[29] Reichel, W., Walter, W., Sturm-Liouville type problems for the p-Laplacian under asymptotic non-resonance conditions, J. Differential Equations 156 (1) (1999), 50-70.

[30] Ruf, B., On nonlinear elliptic problems with jumping nonlinearities, Ann. Mat. Pura Appl. (4) 128 (1981) 133-151. 
[31] Rynne, B., The Fučik spectrum of general Sturm-Liouville problems, J. Diff. Equ. 161 (2000), 87-109.

[32] Schechter, M., The Fučík spectrum, Indiana Univ. Math. J. 43 (4) (1994), 1139-1157.

Dipartimento di Matematica, Universitá di Milano, Via Saldini 50, Milano, Italy E-mail address: bernhard.ruf@unimi.it 